Ada Muellner

Gary M. Glazer

Maximilian F. Reiser

William G. Bradley Jr.

Gabriel P. Krestin

Hedvig Hricak

James H. Thrall

\title{
Advancing radiology through informed leadership: summary of the proceedings of the Seventh Biannual Symposium of the International Society for Strategic Studies in Radiology (IS ${ }^{3} \mathbf{R}$, 23-25 August 2007
}

Received: 16 December 2008

Accepted: 18 January 2009

Published online: 11 March 2009

(C) The Author(s) 2009.

This article is published with open access at Springerlink.com
A. Muellner · H. Hricak $(\bowtie)$

Department of Radiology, Memorial

Sloan-Kettering Cancer Center, 1275 York Avenue, room C-278, New York, NY 10065, USA

e-mail: hricakh@mskcc.org

\section{G. M. Glazer}

Department of Radiology, Stanford

University School of Medicine,

1201 Welch Road, Room P-263,

Palo Alto, CA 94304, USA

M. F. Reiser

Department of Clinical Radiology,

Ludwig Maximilian University,

Nussbaumstr. 20,

80336 Munich, Germany
W. G. Bradley Jr.

Department of Radiology,

University of California, San Diego,

Medical Center,

402 Dickinson Street, Suite 454,

San Diego, CA 92103-8224, USA

G. P. Krestin

Department of Radiology,

Erasmus University Medical Center,

P.O. Box 2040,

3000 CA Rotterdam, The Netherlands

J. H. Thrall

Department of Radiology,

Massachusetts General Hospital,

FND 216, 55 Fruit Street,

Boston, MA, USA

Abstract The International Society for Strategic Studies in Radiology $\left(\mathrm{IS}^{3} \mathrm{R}\right.$ ) brings together thought leaders from academia and industry from around the world to share ideas, points of view and new knowledge. This article summarizes the main concepts presented at the $2007 \mathrm{IS}^{3} \mathrm{R}$ sympo- sium, providing a window onto trends shaping the future of radiology. Topics addressed include new opportunities and challenges in the field of interventional radiology; emerging techniques for evaluating and improving quality and safety in radiology; and factors impeding progress in molecular imaging and nanotechnology and possible ways to overcome them. Regulatory hurdles to technical innovation and drug development are also discussed more broadly, along with proposals for addressing regulators' concerns and streamlining the regulatory process.

Keywords Interventional radiology • Molecular imaging · Device approval processes - Drug approval processes · Health-care quality · Radiology ·

Leadership

\section{Introduction}

The modern transformation of radiology into an essential guiding hand of medical practice began with the invention of computed tomography imaging in the early 1970s and continues today with novel applications based on molecular methods and nanotechnology. The topical agenda of the 7th Biannual Symposium of the International Society for Strategic Studies in Radiology (IS ${ }^{3}$ ), "Advancing Radiology through Informed Leadership," was chosen by Program Committee Chairman Maximilian Reiser to promote exploration and discussion of radiology's continuing transformation, its implications for the care of patients, and, more broadly, its impact on the health-care systems of the world.

Major themes of the Symposium included the increasing role of image-guided therapeutic interventions, the central role of quality and safety, and the emergence of molecular imaging and nanotechnology. Regulatory hurdles for technical innovation were addressed for both drugs and devices.

The IS ${ }^{3} \mathrm{R}$ brings together thought leaders from academia and industry from around the world to share ideas, points of view, and new knowledge. The following summary reflects the contributions of these diverse voices and provides a unique window for looking at important trends that are shaping the future of radiology. 


\section{Interventional radiology: new fields and challenges}

The technology for minimally invasive, image-guided interventions is advancing rapidly, aided by the convergence of innovations from numerous scientific and medical disciplines. As a result, a host of new options for the minimally invasive diagnosis and treatment of disease are broadening the horizons of interventional radiology (IR).

Fusion of images from different radiological techniques is allowing more accurate and targeted intraoperative navigation. For example, preoperative 3D CT angiography is being successfully co-registered with intraoperative $2 \mathrm{D}$ angiography to provide better depth perception and navigation during angiographic liver interventions, with motion correction for breathing [1]. Anatomical imaging is also being combined with functional or molecular imaging. Thus, the use of ultrasound to identify suspicious nodules may be accompanied by the use of a gamma probe to determine whether the nodules display functional activity indicative of cancer [2]. In patients suspected to have prostate cancer, targeted biopsies guided by the fusion of ultrasound with MRI and MR spectroscopy are allowing better cancer detection [3].

"Smart" devices are also improving navigation during image-guided interventions. For example, the insertion of miniature "smart" electromagnetic sensors into interventional tools such as guide wires or needles allows their progress through the body to be followed on a GPS-like tracking system [4]. In this approach, the magnetic space of the patient is co-registered with preoperative 3D imaging data, essentially creating a road map for the intervention. Not only does this lessen the need for intraoperative imaging (which may involve exposure to ionizing radiation and/or contrast media), it also opens up more possibilities for bringing information from sophisticated preoperative imaging techniques - such as PET or MRI - directly into the interventional process [4].

Relatively inexpensive robotic instruments for needle placement are now available. CT-integrated robots place needles more accurately and consistently than humans, and with the use of treatment planning software, they can identify and access the most direct routes to their targets. Although their cost-effectiveness has not yet been proven in large-scale studies, they work rapidly and also reduce interventional radiologists' exposure to radiation [5].

Further tailoring of interventions is becoming possible through the advent of "smart" drugs that are designed to complement interventional techniques [6]. One example is Thermodox ${ }^{\mathrm{TM}}$, which consists of thermosensitive liposomal capsules that contain the chemotherapeutic agent doxorubicin. The capsules release the doxorubicin only under the high temperatures induced by thermal ablation. When the capsules are injected into the tumor vasculature at the time of radiofrequency ablation, the doxorubicin takes effect throughout the tumor, including at the edges, where radiofrequency ablation alone tends to be ineffective.
Because the doxorubicin is released only in the heated area, its toxic side effects are limited. Another concept consists of combining the application of thermosensitive liposomal capsules with regional hyperthermia induced by noninvasive, image-guided focused ultrasound [7].

With advances in nanotechnology, the potential for the development of "smart" drugs appears limitless. For instance, targeting antibodies or ligands could be attached to drug-carrying capsules made with bound metal (to allow imaging). Not only the ligands, but the agents inside the capsules could be varied to target specific disease characteristics. In a new paradigm of "molecular IR," molecular imaging-guided interventions could be used to determine the molecular characteristics of a disease and to subsequently deliver the appropriate targeted therapy [7].

Emerging cellular therapies could also play a role in IR. Preliminary research suggests that the insertion of autologous bone marrow cells after myocardial infarction can improve left ventricular function and myocardial viability [8]. Various imaging techniques, including MRI, PET, and SPECT, have been used to assess the functional effects of cell therapy, and methods for tracking and labeling cells with radionuclides and superparamagnetic agents are being developed [9]. Although extensive clinical trials are still needed, cellular therapy could potentially become an important alternative to heart transplantation for the vast numbers of patients suffering from severe heart disease [10].

MR-guided focused ultrasound (MRgFUS) is another novel interventional technique that could have applications in a multitude of patients. In this procedure, hundreds of ultrasound beams are directed at a focal point, where they cause intensive local heating and tissue destruction. The entire procedure, which is performed on an outpatient basis, takes place inside the bore of the MRI magnet. MR imaging is used to guide the beam, avoiding critical structures such as nerves, scar tissue, surgical clips, and bowels, and MR thermal mapping is used to monitor the temperature rise (through a phase shift) and tissue destruction [11]. MRgFUS is gaining acceptance as a non-invasive treatment for uterine fibroids [12]. In a study conducted in the UK, 80/100 patients treated with MRgFUS for uterine fibroids had significantly less severe symptoms 6 months after treatment [13]. Data suggest that MRgFUS may have more long-lasting effects in the treatment of uterine fibroids than the more invasive alternative of myomectomy. MRgFUS has also been used for the palliative treatment of bone metastases. Destruction of periosteal nerves has been suggested as a possible explanation for the analgesic effect, which cannot be explained simply by the avid absorption of ultrasound waves by bone. Although the inability of ultrasound waves to pass beyond bones limits the areas that can be directly accessed by the technique, MRgFUS is now being evaluated for the treatment of intracranial tumors. Even though $90 \%$ of the energy is reflected back by the 
calvarium, enough power remains to thermally ablate a glioblastoma multiforme or liquefy a clot in acute stroke. (The reflected energy is absorbed by a water bag.) Moreover, results in the treatment of breast cancer as well as soft-tissue metastases using MRgFUS have been reported [14]. Radiologists have an essential role to play in the development and application of MRgFUS, as it requires substantial skill in the use of imaging to guide the ultrasound beam.

The developments described above suggest great potential for the growth of IR. However, despite a history of introducing innovative and widely applicable techniques, the field of IR has grown at a very slow rate, such that in 2002 there were only around 200 IR training positions in the United States (as compared to around 2,300 in cardiology the year before) [15]. To some leaders in the field, these numbers suggest that the very survival of IR is at risk [16].

Repeatedly, techniques that have started out in IR have ended up being adopted by organ-based specialties, and the referrals to IR for these procedures have dropped off. For example, by 2003, cardiologists in the US had caught up with radiologists in the performance of peripheral vascular stent procedures, and now the majority of such procedures are performed by cardiologists [17]. With their direct access to patients, their comparatively large numbers of personnel, and their greater financial resources and research activity, organ-based specialties have tremendous advantages over IR in the competition for patients [18].

To sustain and enhance the relevance of IR, adjustments to the standard IR training may be needed. Arguably, more of the training could be focused on IR procedures and equipment and less on diagnostic imaging, while still providing the core skills necessary to achieve board certification in radiology. Clinical skills should be emphasized. Efforts should also be made to increase recruitment into IR training programs, perhaps by looking for candidates outside the field of radiology, in specialties that attract more physicians who seek patient contact [19]. The move in Europe to a 3 -year core training with 2 years devoted to sub-specialty training should help in this regard [20].

The relationship of IR to radiology may need to be reconsidered. Should IR be separated from radiology and become an independent clinical specialty? Should it remain within radiology? Or should it be organized into partnerships with separate organ-based specialties? Each scenario has advantages and disadvantages [21]. One advantage of remaining within radiology is the access this affords to advanced imaging equipment. However, if IR is to stay within radiology, the differences between the work of interventional radiologists and that of diagnostic radiologists must be addressed. For example, since the former cannot bill as many procedures as the latter, they should perhaps have a separate accounting system. More hospital and departmental resources should be devoted to marketing and community outreach for IR. In addition, regardless of which organizational approach is pursued, interventional radiologists should seek to obtain the status of true clinicians, with admitting privileges and the administrative support and infrastructure that will allow them to take more direct responsibility for patient care.

Interdisciplinary collaboration and involvement in research are also essential to enhance the growth of IR. At present, IR lags far behind organ-based specialties in the publication of original research. Many promising new interventional techniques (including most of those described above) have yet to be validated through extensive clinical trials. More studies are needed to demonstrate their benefits to patient outcomes [22] as well as to workflow. With the continual advancement of technology, there are also endless possibilities for creating new interventional approaches. To enhance their contributions to research, interventional radiologists should build strong collaborative ties with physicians in other specialties as well as with the radiochemists, physicists, computer scientists, engineers, and basic scientists whose knowledge is essential for developing new minimally invasive techniques.

Regardless of whether they continue to be applied under the rubric of IR, the skills of interventional radiologists are going to be increasingly in demand [23]. Furthermore, the interventional radiologist who can deliver a comprehensive range of emergency procedures will continue to be held in high regard by professional colleagues.

\section{Quality and safety}

Rising health-care costs in the developed world have led to increased scrutiny of the quality of care being purchased. Four broad components of quality health care are now widely recognized: appropriateness of care (as opposed to overuse, underuse, or misuse of procedures); coordinated care that links providers and information; safe, error-free care; and patient-centered, timely care [24]. The methods for evaluating these aspects of quality, however, are still being developed and debated.

One common way of gauging quality has been to compare usage of medical procedures across geographic regions, institutions, and individual providers. For years, wide variations in usage have been acknowledged to exist in the US [25]. However, solutions to this problem have been slow in coming. As recently as 2003, McGlynn et al. found that on average, Americans received only about half of recommended medical care (as defined by a panel of physicians working on the study) [26]. Underuse of medical processes was a much greater problem than overuse, with $46.3 \%$ of study participants not receiving recommended care and $11.3 \%$ receiving superfluous and potentially harmful care.

Within radiology, it appears that the opposite may be true. A study of the health-care provided to employees of General Electric suggested that approximately half $(\$ 60$ million-worth) of the radiological tests ordered were 
unnecessary [27]. Nevertheless, when used appropriately radiological services can make health care better, quicker, and cheaper, most often by diminishing diagnostic uncertainty and obviating the need for additional (and more invasive) procedures. More studies are needed to demonstrate that radiology can improve outcomes and reduce costs at every level, from the clinician, to the department, the hospital, the insurer, the community, and the nation $[28,29]$.

Refinements in the criteria for test usage and reporting can lead to considerable increases in cost-effectiveness. For example, a study in the UK found that the incremental cost of using breast MRI would be $£ 28,284$ per cancer detected [30] if it were applied in all patients who underwent mammography, but only $£ 11,731$ if it were applied in place of mammography for patients with BRCA1 and $£ 15,302$ if it were applied in addition to mammography in patients with BRCA2 [30]. Partly as a result of such findings, the use of MRI in patients at high risk for breast cancer is now being funded by the National Health Service (NHS) [31]. Another study found that by not reporting lesions smaller than $6 \mathrm{~mm}$ seen on CT colonography, radiologists could substantially reduce costs and complications while producing only a trivial decrease in cancer detection [32].

To help develop imaging recommendations, more studies are needed to determine whether imaging tests are likely to improve clinical decision-making and outcomes. Modeling can be used for this purpose. To yield results that are applicable to a broad spectrum of clinical settings, more meta-analyses of small studies, as well as more large multicenter studies should be done. Aids for designing and implementing clinical trials of imaging are readily available. For example, the Web site of the American College of Radiology Imaging Network provides access to standard enrollment form templates and sample protocols [33].

Decisions about cost-effective radiological test usage may be complicated by concerns about exposure to ionizing radiation. Increasingly, patients are becoming aware of the risks of radiation exposure. In the late 1990s, the European Community issued Directive 97/43/EURATOM outlining the need for justification, optimization, and auditing of the use of ionizing radiation in health care. Over the past 5 years, the European Commission issued European Guidelines for Multislice Computed Tomography (an update of an earlier set of guidelines for the use of CT) [34] and launched the project "Safety and Efficacy of Computed Tomography: A Broad Perspective." The latter project brings together radiologists and physicists from leading European academic institutions to study CT justification and radiation dose optimization and measurement and to design practical tools for improving practice (e.g., clinical decision trees and optimized CT techniques). The project has resulted in published papers, conference presentations, and a report to the Commission. However, dissemination of the information has been incomplete. Research is being outpaced by advances in imaging technology. Furthermore, as CT is now widely available, relatively affordable, and quick, guidelines for limiting its use are being ignored to meet the demand for imaging, and it appears that many, perhaps even most, CT examinations performed in Europe are being requested in advance of patients' needs [35].

Increasing the availability of both ultrasound and MRI should reduce reliance upon techniques involving X-rays, particularly for young patients [36]. To address the problem of radiation exposure more effectively, the government must continue to support research and not only pass, but also enforce laws through auditing. In addition, researchers must work closely with industry to ensure that quality and safety measures are incorporated into technology development, and leaders in government, health care and industry must prioritize quality and safety above (real or imagined) productivity.

Various approaches are being pursued for improvingand not just assessing - the quality and appropriateness of health care. For patients on Medicare in the US, efficiency measures have been proposed for the use of certain radiological examinations, including MRI of the lumbar spine and mammography. Recall rates after mammograms are being evaluated to determine whether mammograms are being performed in the appropriate patients [37]. Meanwhile, private insurers have begun to scrutinize utilization rates per thousand patients. Ultimately, to bring about large-scale changes in practice, information on performance at all levels will need to be made routinely available - a process that will require substantial changes in health information systems, including automated entry of essential data for clinical decision-making [24].

Health-care accreditation systems are now in use in the US, Finland, and Australia. In 2006, the Royal College of Radiologists (RCR) and the Society and College of Radiographers began to develop the Radiology Accreditation Project (RAP) to assess radiological services in the UK [38]. At present, the RAP is still in the pilot stage and is voluntary. Accreditation requires demonstration of appropriate protocols for monitoring and assuring the quality of service relative to published standards for safety, patient experience, clinical performance, and utilization of resources and workforce. It also requires the development of a quality improvement plan with achievable goals. Both self-assessment and assessment by independent auditors are performed.

The RCR is also working to establish a system for relicensing and re-certification of radiologists in the UK, with the goal of identifying poorly performing ones. It is known that the performance of approximately $2 \%$ of radiologists in the UK falls 2 standard deviations below the mean, yet only $0.5-1 \%$ of radiologists are referred for evaluation by the National Clinical Assessment Service [39]. Measuring the overall performance of individual radiologists can be tricky. Theoretical knowledge and, to some extent, practical skills can be assessed by examinations 
or more complex tests. However, assessment of professionalism and behavior has generally relied on informal feedback, which is often considered unreliable and ignored. In a survey that asked RCR members and fellows to choose the best methods for identifying poorly performing radiologists, third place went to peer review of cases, and second place went jointly to attendance at discrepancy/error/ complications meetings and on-line assessments linked with educational programs. First place went to a less conventional approach: multiple-source feedback, consisting of a confidential questionnaire for peers and patients, ranking of behavior, skills and knowledge, and computerized comparison of the radiologist's scores with those of other radiologists and doctors, ideally both nationally and locally. Rather than the assignment of pass or fail marks, the proposed multi-source feedback approach requires further evaluation of any radiologists who receive consistently low scores.

Although much emphasis is now being placed on increasing efficiency, it is important to keep the focus of attention on the patient. Business objectives are best met by meeting patient care objectives. For example, the business objective of an inpatient unit is to reduce the length of the average patient stay in the hospital; for radiology, this means minimizing the turnaround time from study request to study completion, which both shortens the patient's stay and improves the patient's care [40]. The use of flow charts in work process analyses can help identify ways to reduce waste and the potential for error. Some of the solutions may be non-intuitive. For example, net savings may result from hiring an extra full-time technician to keep an expensive piece of equipment (e.g., an MRI machine) in operation during examination preparation times and the primary technician's lunch hour and coffee breaks. Conversely, for equipment that is less expensive to purchase and operate, it may be more cost-effective to buy many units, so that they can readily be used whenever needed, even if they are not constantly in use. Improving productivity and care requires establishing the right balance between equipment and staff and providing the necessary training for staff to make optimal use of equipment [41].

At present, the error rate in the US health-care industry far exceeds those in the airline, telecommunications, and computer industries. Teamwork and communication are essential for improving this state of affairs. Traditionally, the culture in medicine has encouraged physician autonomy over teamwork. However, physicians and staff with average skills but an exceptionally well-designed work process will outperform those with better skills but a less coordinated work process [42]. Approaches used to encourage communication in the airline industry, such as anonymous reporting of errors and elimination of penalties for errors, have been applied in health-care organizations and have been found to increase the rate of error reporting, stimulate discussions to improve work processes, and ultimately decrease the incidence of errors [43]. Multidisciplinary team meetings allow radiologists to make recommendations for the clinically relevant use of imaging and deliver clinically relevant interpretations.

Information technology, such as decision support for order entry and computer-aided diagnosis algorithms, can also help reduce errors and improve quality. Furthermore, teleradiology is increasingly being used around the world to augment local radiological services $[44,45]$. In the US, the most common use of teleradiology has been to allow radiologists to interpret images from home, but approximately $50 \%$ of radiological practices are now using outside service providers for interpretations on nights and weekends. In parts of Europe and Asia, teleradiology is being used across borders either to serve areas with a shortage of radiologists or to allow secondary consultations with specialists, or both [46]. The use of Indian teleradiology services in Singapore has improved care by reducing patient wait times and allowing more rapid clinical decision-making; it has also forced radiologists in Singapore to work faster in order to prove their worth.

Limited communication may be the most significant drawback of teleradiology, as direct access to complete clinical information, including prior imaging studies, is rarely available, and teleradiology reports are usually submitted by e-mail or fax, without direct contact between the radiologist and the referring physician. Auditing is needed to ensure that teleradiologists meet the same certification and training requirements as local radiologists and that adequate clinical and technical standards are being maintained. Although a study comparing interpretations made by NHS radiologists with those made by teleradiologists at an independent service provider found few discrepancies, most of the cases examined were of low complexity [47]. The RCR, the American College of Radiology, and the European Society of Radiology (in conjunction with the European Union of Medical Specialists) have all issued fairly similar guidelines for quality assurance in teleradiology [48].

\section{Molecular imaging and nanotechnology}

The potential of molecular imaging to revolutionize health care is often spoken of in rhapsodic terms. Yet the practice of molecular imaging (MI) within radiology continues to be confined to a relatively small number of institutions and settings. Though MI already has clear applications in neurodegenerative conditions, cardiovascular disease, inflammation [49], and above all, cancer, a number of obstacles are impeding progress toward its broad clinical implementation-some of them avoidable, others less so.

It is hoped that "system characterization" by imaging probes will ultimately allow molecular knowledge developed in the laboratory to be translated to clinical disease management [50]. In combination with in-vitro molecular markers, MI (performed mainly with multimodality techniques, such as PET/CT and PET/MR) [51] should 
facilitate earlier, even preclinical disease detection and characterization, prediction of prognosis, targeted treatment, and precise treatment follow-up, allowing "personalized medicine" that is tailored closely to the individual patient. However, both physical and technical factors make achieving this vision a highly complex and challenging endeavor. The body is equipped with many barriers to molecular transport - such as nuclear, cellular, and vessel walls, and the blood brain barrier. The development of probes suitable for use in humans is limited by pharmacokinetics, biocompatibility, and toxicity concerns, and imaging speed and resolution. Contrast agents and radiopharmaceuticals must have sufficient half lives and must aggregate in sufficient concentrations to allow effective imaging once they have reached their target destinations. In addition, for any one disease, a limited number of overexpressed molecular markers will exist for targeting [52].

Molecular interactions and disease processes are complex, and knowledge of them remains limited. Although MI with PET has been used successfully to explore how new drugs interact with targeted systems and to narrow the feasible dose ranges, PET imaging results can also be misleading. For example, during its development, the antipsychotic drug aripiprazole had no clinical effectiveness at the feasible dose range identified by PET, but in practice, the drug was found to be effective and safe at substantially higher doses. While MI can show on-target biological activity and provide essential information about the biodistribution of drugs, both more knowledge and more probes are needed to increase the reliability of MI for assessing drug effects. In certain instances, it may soon be possible for MI to play a role in disease stratification, patient selection for therapeutic agents, and dose selection [52], but MI is unlikely to replace clinical endpoints in drug development for a long time to come.

Nanotechnology offers enormous versatility in the development of MI probes. The advent of particle replication in non-wetting templates (PRINT) technology has made it possible to mold nanoparticles small enough to cross the blood-brain barrier [53]. Not only the size, but also the ability of nanoparticles to deform can be tailored to their purpose, increasing or limiting their uptake. In addition, nanoparticles can be filled with cargoes (e.g., antibodies) and can have entities (e.g., targeting ligands) attached to their surfaces [54]. Applications for which nanoparticle-based contrast agents are expected to be introduced into clinical practice within the next 10 years include imaging of metastatic lymph nodes, vulnerable atherosclerotic plaque imaging, macrophage imaging for inflammatory and degenerative diseases (e.g., multiple sclerosis, polyarthritis and osteomyelitis), and imaging of amyloid plaques in Alzheimer's disease.

Many more probes might now be on the horizon were it not for the vast amounts of time and money required to overcome regulatory hurdles. In the US, this problem has been exacerbated by misconceptions about regulatory requirements. Many applications for pharmacology/ toxicology studies have been submitted with much more background data than necessary [55]. In 2006, the FDA issued guidelines for exploratory investigational new drug (IND) studies (e.g., screening studies or microdose studies) that are conducted early in phase 1 of the trial process, involve very limited human exposure for a short time period (e.g., 7 days), and have no diagnostic intent [56]. The guidelines explain that because exploratory IND studies present fewer potential risks than do traditional phase 1 studies that look for dose-limiting toxicities, they can be initiated with less, or different, preclinical support than is required for traditional IND studies [56]. Exploratory IND studies allow a large number of agents to be tested rapidly in order to distinguish the promising from the not-so-promising, decreasing the likelihood of failure in later clinical trials.

While exploratory IND studies are extremely useful, further simplification of regulatory processes is necessary, as is the creation of a viable business model for probe development. The development of MI probes that deliver drugs could allow much more targeted treatments of disease with less morbidity. However, trials of such probes are especially complex and doubly risky for the sponsors, since either the imaging probe or the drug may fail. Collaboration between academia and industry in probe development has been hampered by concerns about intellectual property rights. Furthermore, lack of reimbursement discourages experimental use of molecular imaging in hospital settings, particularly since the required multimodality imaging techniques are expensive [57]. Despite these obstacles, collaboration between industry and academia is possible in MI research and probably essential for academic institutions. In a recent survey of academic radiology departments with MI programs in the US, only $16.1 \%$ had no alliances with industry [58].

Another barrier to progress in the clinical implementation of MI is the need for expertise from multiple disciplines. Radiologists tend to have little understanding of molecular biology, and few have any familiarity with molecular imaging, since it is not included in standard clinical training. To encourage more radiologists (and other physicians) to become involved in the field, MI rotations should be created, and more MI fellowships should be offered. In the survey referenced above, respondents identified staff training and recruitment as the most essential elements for success [58]. In light of the extensive training in both basic science and multimodality imaging required for the field, the creation of a new specialty of MI may be warranted, with the possibility of dual boarding in diagnostic radiology and MI, or nuclear medicine and MI.

Because MI involves not only multiple disciplines but multiple kinds of technology, its success will require major investments in human resources and infrastructure. It will also require significant changes in organizational structures 
and the setting aside of turf battles. Conceivably, molecular biology, pathology, and imaging could be combined under one umbrella, perhaps that of "biomedical imaging" or "imaging science" [60]. Alternatively (or concomitantly), the typical "Department of Diagnostic Radiology" could be replaced by a "Department of Diagnostics," combining pathology with radiology and MI, and using pathological analysis of genes and proteins to identify targets and create agents for MI in individual patients. Regardless of the specific restructuring plan chosen, broad clinical implementation of MI will depend on collaboration, co-training, and co-ownership.

\section{Regulatory hurdles}

Approval hurdles for pharmaceutical media and technology

Regulatory requirements pose daunting barriers to the introduction of radiological innovations. For example, a pharmaceutical company is likely to spend over $\$ 300$ million to bring a new contrast agent to market, in a process that typically takes about 9 years. And that is only the beginning. For each new indication, about $\$ 15$ million more in funds is required and around 5 more years, although most radiologists ignore the package insert and engage in "off label" use. While the process is somewhat less onerous in Europe than in the US, regulatory hurdles appear to be increasing around the world.

The need to meet different regulatory requirements in different areas of the world adds to the complexity and costs of the process. Currently, regulations differ in the EU and the US, China and Japan, with the regulatory policies of the latter two countries being closer to those of the US. In both the EU and the US, regulators look for accuracy (e.g., as measured by sensitivity and specificity), reliability, clinical value, and a favorable risk/benefit ratio. However, far more contrast agents have been approved in Europe than in the US to date. This may be partly because US regulators seem to place a greater emphasis on the demonstration of $\mathrm{p}$-values showing statistically significant increases in value over existing technology or agents, whereas EU regulators are more content to see any demonstration of utility, even if it is only for a secondary aim [61]. Furthermore, in the US, the requirements are often not aligned with the intended use of the agent or device. For example, even if the manufacturer's goal for a contrast agent is to increase tumor visualization, the FDA may demand that the manufacturer also demonstrate an improvement in tumor diagnosis. Such a demand may prevent a useful agent from coming to market, or may require it to be tested (and approved) for a narrower cohort and indication.
Advances in technology occur faster than does the development of new contrast agents. Thus, delays in the approval process for an agent can make the agent or the application tested obsolete by the time it becomes available. Moreover, partly because of the fast pace of technological change, standardization of imaging protocols in multi-center trials is difficult, as is comparison of the results of multiple single-center studies.

In a phenomenon sometimes referred to as "regulatory creep," the FDA has repeatedly come back to manufacturers and pharmaceutical companies with requests for more data, more details, and stricter adherence to protocols. To prevent this, the FDA should be required to make binding agreements about what is required at the beginning of the regulatory process. Recently, the FDA began to call for clinical trials to assess the use of a contrast agent on a new imaging platform, even when the contrast agent has already been approved. This shortens the effective lifetime of the new device, reducing the incentive for the manufacturer to invest in the trial process.

In Japan, regulatory requirements have also multiplied recently. The average approval time for a new medical device in Japan in 2004 was 1,083 days, compared to 356 in the US [62]. The number of new device applications submitted by US companies for review by Japanese regulators fell from 132 in 2003 to only 8 in $2005-a$ decrease of $94 \%$. Representatives of the US medical technology industry cited "burdensome applications and an unpredictable approval process" as reasons for the decline [62].

In the US, it appears that increasing delays in the approval process are partially due to a lack of trained regulatory personnel, as well as regulators' tendency to become concerned by negative anecdotal studies. In Japan, where the approval process is even more complex, the number of experienced reviewers is even smaller, and there is a large backlog of applications. It may seem curious that regulations should be multiplying even during times of reviewer staffing shortages. Some speculate that the changes may be part of a deliberate effort on the part of governments to reign in health-care spending by reducing the approval of new medical devices and drugs. However, such a practice may in fact lead to greater social and economic costs overall. Increasing the duration and cost of the regulatory process is only likely to increase the cost of a medical device or agent once it comes to market. Furthermore, improvements in technology ultimately lower the cost of quality health care. For example, since CT was first introduced, the number of CT slices per second has increased-from 1 in 1995 to 387 in 2006; meanwhile, the cost per slice has gone down, so that for the same overall price, much more information is acquired. While it is easy to add up medical expenses, it is less easy to calculate the costs (not to mention the ethical drawbacks) of not delivering efficient, highquality medical care. 
Proposals for improvements

To speed up the introduction of innovations into clinical practice, regulatory processes should be harmonized globally. Members of academia and industry should encourage their governments to adopt the recommendations of the Global Harmonization Task Force, a partnership between regulatory authorities and regulated industry representatives from the European Union, the US, Canada, Australia, and Japan.

The development of new medical products could be streamlined through increased collaboration between industry and academia. For example, the pattern in technology development has been to design a piece of equipment and then search for ways to apply it clinicallya rather inefficient process. More communication between members of academia and industry could allow technology to be developed in response to specific clinical needs [63]. Fellowships enabling representatives of academia to train with industry (or vice versa) could lead to greater mutual understanding of priorities and could help improve multicenter clinical trials - say, by facilitating standardization of technology and of institutional imaging protocols.

Efficiency in product development could also be improved by considering the whole imaging system, rather than working on different parts of it in isolation. Modern imaging requires a series of steps, including data acquisition, data reconstruction, and processing, data analysis and output, and data interpretation. Different disciplines are involved in the design of the equipment used for the separate steps, including engineering, physics, and computer science. Greater communication among all disciplines involved is more likely to result in advances that improve the effectiveness of the overall imaging system [63].

Trial design and conduct could also be improved with more collaboration among industry, academia, and government agencies - e.g., through organizations such as the American College of Radiology Imaging Network (ACRIN) [64]. Curricula could be redesigned to mandate training in basic and clinical research all along the educational pathway, starting with medical school. In addition, industry and academia could work together to train radiologists in the use of new imaging technology and applications even before they receive regulatory approval. This could not only improve the performance of new devices or techniques in clinical trials, but also could lead to earlier, broader acceptance of the techniques and faster progress toward reimbursement.

Acknowledgments The authors thank Adrian K. Dixon, MD, for his valuable editorial advice.

Open Access This article is distributed under the terms of the Creative Commons Attribution Noncommercial License which permits any noncommercial use, distribution, and reproduction in any medium, provided the original author(s) and source are credited.

\section{References}

1. Groher M, Jakobs TF, Padoy N, Navab N (2007) Planning and intraoperative visualization of liver catheterizations: new CTA protocol and 2D-3D registration method. Acad Radiol 14:1325-1340

2. Wendler T, Feuerstein M, Traub J, Lasser T, Vogel J, Daghighian F, Ziegler SI, Navab N (2007) Real-time fusion of ultrasound and gamma probe for navigated localization of liver metastases. Med Image Comput Comput Assist Interv Int Conf Med Image Comput Comput Assist Interv 10(Pt 2):252-260

3. Singh AK, Kruecker J, Xu S, Glossop N, Guion P, Ullman K, Choyke PL, Wood BJ (2008) Initial clinical experience with real-time transrectal ultrasonography-magnetic resonance imaging fusion-guided prostate biopsy. BJU Int 101:841-845
4. Wood BJ, Zhang H, Durrani A, Glossop N, Ranjan S, Lindisch D, Levy E, Banovac F, Borgert J, Krueger S, Kruecker J, Viswanathan A, Cleary K (2005) Navigation with electromagnetic tracking for interventional radiology procedures: a feasibility study. J Vasc Interv Radiol 16:493-505

5. Fichtinger G, DeWeese TL, Patriciu A, Tanacs A, Mazilu D, Anderson JH, Masamune K, Taylor RH, Stoianovici D (2002) System for robotically assisted prostate biopsy and therapy with intraoperative CT guidance. Acad Radiol 9:60-74

6. Miraglia R, Pietrosi G, Maruzzelli L, Petridis I, Caruso S, Marrone G, Mamone G, Vizzini G, Luca A, Gridelli B (2007) Efficacy of transcatheter embolization/chemoembolization (TAE/TACE) for the treatment of single hepatocellular carcinoma. World J Gastroenterol 13:2952-2955
7. Dromi S, Frenkel V, Luk A, Traughber B, Angstadt M, Bur M, Poff J, Xie J, Libutti SK, Li KC, Wood BJ (2007) Pulsed-high intensity focused ultrasound and low temperature-sensitive liposomes for enhanced targeted drug delivery and antitumor effect. Clin Cancer Res 13:2722-2727

8. Strauer BE, Brehm M, Zeus T, Köstering M, Hernandez A, Sorg RV, Kögler G, Wernet P (2002) Repair of infarcted myocardium by autologous intracoronary mononuclear bone marrow cell transplantation in humans. Circulation 106:1913-1918

9. Mowat P, Franconi F, Chapon C, Lemaire L, Dorat J, Hindré F, Benoit JP, Richomme P, Le Jeune JJ (2007) Evaluating SPIO-labelled cell MR efficiency by three-dimensional quantitative T2* MRI. NMR Biomed 20:21-27 
10. Gowdak LH, Schettert IT, Baptista E, Lopes NL, Rochitte CE, Vieira ML, Grupi CJ, César LA, Krieger JE, de Oliveira SA (2008) Intramyocardial injection of autologous bone marrow cells as an adjunctive therapy to incomplete myocardial revascularizationsafety issues. Clinics 63:207-214

11. Hengst SA, Ehrenstein T, Herzog H, Beck A, Utz-Billing I, David M, Felix R, Ricke J (2004) Magnetic resonance tomography guided focussed ultrasound surgery (MRgFUS) in tumor therapy-a new noninvasive therapy option. Radiologe 44:339-346

12. Hindley J, Gedroyc WM, Regan L, Stewart E, Tempany C, Hynyen K, Mcdannold N, Inbar Y, Itzchak Y, Rabinovici J, Kim HS, Geschwind JF, Hesley G, Gostout B, Ehrenstein T, Hengst S, Sklair-Levy M, Shushan A, Jolesz F (2004) MRI guidance of focused ultrasound therapy of uterine fibroids: early results. AJR Am J Roentgenol 183:1713-1719

13. Stewart EA, Rabinovici J, Tempany CM, Inbar Y, Regan L, Gostout B, Hesley G, Kim HS, Hengst S, Gedroyc WM (2006) Clinical outcomes of focused ultrasound surgery for the treatment of uterine fibroids. Fertil Steril 85:22-29

14. Furusawa $H$, Namba K, Nakahara $H$, Tanaka C, Yasuda Y, Hirabara E, Imahariyama M, Komaki K (2007) The evolving non-surgical ablation of breast cancer: MR guided focused ultrasound (MRgFUS). Breast Cancer 14:55-58

15. Licurse A, Saket DD, Sunshine JH, Maynard CD, Forman HP (2006) Update on the diagnostic radiology employment market: findings through 2005. AJR Am J Roentgenol 187: W249-W254

16. Adam A (2006) The 2006 Charles T. Dotter lecture: interventional radiology-veni, vidi, vanished. J Vasc Interv Radiol 17:1399-1403

17. Levin DC, Rao VM, Parker L, Bonn J, Maitino AJ, Sunshine JH (2005) The changing roles of radiologists, cardiologists, and vascular surgeons in percutaneous peripheral arterial interventions during a recent five-year interval. J Am Coll Radiol 2:39-42
18. Levin DC, Rao VM, Bonn J (2005) Turf wars in radiology: the battle for peripheral vascular interventions. J Am Coll Radiol 2:68-71

19. Nicholson AA, Adam A (2006)

"Whatever happened to the class of 2000 ?" An outcome survey of potential interventional radiologists. Clin Radiol 61:706-709

20. European Society of Radiology (2005) Detailed Curriculum for Subspecialty Training. http://www.escr org/downloads/VI_European

Training Charter.pdf. Accessed 22 Feb 2008

21. Becker GJ (2001) 2000 RSNA annual oration in diagnostic radiology: The future of interventional radiology. Radiology 220:281-292

22. Molyneux AJ, Kerr RS, Yu LM, Clarke M, Sneade M, Yarnold JA, Sandercock P (2005) International Subarachnoid Aneurysm Trial (ISAT) Collaborative Group. International subarachnoid aneurysm trial (ISAT) of neurosurgical clipping versus endovascular coiling in 2143 patients with ruptured intracranial aneurysms: a randomised comparison of effects on survival, dependency, seizures, rebleeding, subgroups, and aneurysm occlusion. Lancet 366:809817

23. Woolfson JP, McLaren K, Baerlocher MO (2007) Shortcomings in interventional radiology in Canada and what is needed for change. J Vasc Interv Radiol 18:1404-1408

24. Commonwealth Fund National Scorecard on U.S. Health System Performance (2006) http://www.cmwf. org. Accessed December 10, 2007

25. Landon BE, Normand SL, Frank R, McNeil BJ (2005) Characteristics of medical practices in three developed managed care markets. Health Serv Res 40:675-695

26. McGlynn EA, Asch SM, Adams J, Keesy J, Hicks J et al (2003) The quality of health care delivered to adults in the United States. N Engl J Med 348:2635-2645

27. de Brantes F (2003) Bridges to excellence: a program to start closing the quality chasm in healthcare. J Healthc Qual 25:2-11

28. Kielar AZ, El-Maraghi RH, Carlos RC (2007) Health-related quality of life and cost-effectiveness analysis in radiology. Acad Radiol 14:411-419

29. Mackenzie R, Dixon AK (1995)

Measuring the effects of imaging: an evaluative framework. Clin Radiol $50: 513-518$
30. Griebsch I, Brown J, Boggis C et al (2006) UK Magnetic Resonance Imaging in Breast Screening (MARIBS) Study Group. Costeffectiveness of screening with contrast enhanced magnetic resonance imaging vs X-ray mammography of women at a high familial risk of breast cancer. $\mathrm{Br} \mathrm{J}$ Cancer 95:801-810

31. National Institute for Health and Clinical Excellence (NICE) (2006) Familial breast cancer. The classification and care of women at risk of familial breast cancer in primary, secondary and tertiary care. http://www.nice.org.uk/ nicemedia/pdf/CG41fullguidance.pdf. Accessed 22 Feb 2008

32. Pickhardt PJ, Hassan C, Laghi A et al (2007) Cost-effectiveness of colorectal cancer screening with computed tomography colonography: the impact of not reporting diminutive lesions. Cancer 109:2213-2221

33. Pisano ED, Gatsonis CA, Yaffe MJ, Hendrick RE, Tosteson AN, Fryback DG, Bassett LW, Baum JK, Conant EF, Jong RA, Rebner M, D'Orsi CJ (2005) American College of Radiology Imaging Network digital mammographic imaging screening trial: objectives and methodology. Radiology 236:404-412

34. Bongartz G, Golding SJ, Jurik AG et al (2004) European Guidelines for Multislice Computed Tomography. Funded by the European Commission. Contract number FIGM-CT2000-20078-CT-TIP. http://www.msct.eu. Accessed 28 Feb 2008

35. Golding SJ (2005) Multi-slice computed tomography (MSCT): the dose challenge of the new revolution. Radiat Prot Dosimetry 114(1-3):303-307

36. McNally EG, Wilson DJ, Ostlere SJ (2001) Limited magnetic resonance imaging in low back pain instead of plain radiographs: experience with first 1000 cases. Clin Radiol 56:922-925

37. Woodard DB, Gelfand AE, Barlow WE, Elmore JG (2007) Performance assessment for radiologists interpreting screening mammography. Stat Med 26:1532-1551

38. Radiology Accreditation Program Web site. http://accreditation.rcr.ac.uk. Accessed 22 Feb 2008

39. UK National Clinical Assessment Service. http://www.ncas-resource. npsa.nhs.uk/?EntryId13=9106\&cord $=$ DESC\&char=R. Accessed 22 Feb 2008 
40. Thrall JH (2007) Changing relationships between radiologists and hospitals. Part I. Background and major issues. Radiology 245:633-637

41. Thrall JH (2008) Changing relationships between radiologists and hospitals. Part II. Contracts and resolution of issues. Radiology 246:343-347

42. Swensen SJ (2007) Reliably better, faster, and cheaper soufflés. J Am Coll Radiol 4:86-87

43. FitzGerald R (2005) Radiological error: analysis, standard setting, targeted instruction and teamworking. Eur Radiol 15(8):1760-1767

44. Thrall JH (2007) Teleradiology. Part I. History and clinical applications. Radiology 243:613-617

45. Thrall JH (2007) Teleradiology. Part II. Limitations, risks, and opportunities. Radiology 244:325-328

46. Cheng LT, Ng SE (2006) Teleradiology in Singapore-taking stock and looking ahead. Ann Acad Med Singap 35:552-556

47. Dixon A, Webster P (2007) Outsourced imaging solutions - meeting increasing exam targets in the UK. Imaging management 7:16-17

48. European Society of Radiology (2006) Teleradiology in the European Union http://www.myesr.org/html/img/pool/ ESR 2006 VII Telerad Summary Web.pdf. Accessed 22 Feb 2008

49. Biswal S, Resnick DL, Hoffman JM, Gambhir SS (2007) Molecular imaging: integration of molecular imaging into the musculoskeletal imaging practice. Radiology 244:651-671
50. Stahl A, Wieder H, Piert M, Wester HJ, Senekowitsch-Schmidtke R, Schwaiger M (2004) Positron emission tomography as a tool for translational research in oncology. Mol Imaging Biol 6:214-224

51. von Schulthess GK, Steinert HC, Hany TF (2006) Integrated PET/CT: current applications and future directions. Radiology 238:405-422

52. Eckelman WC, Reba RC, Kelloff GJ (2008) Targeted imaging: an important biomarker for understanding disease progression in the era of personalized medicine. Drug Discov Today 13:748759

53. Wang L, O'Donoghue MB, Tan W (2006) Nanoparticles for multiplex diagnostics and imaging. Nanomed $1: 413-426$

54. Gratton SE, Ropp PA, Pohlhaus PD, Luft JC, Madden VJ, Napier ME, Desimone JM (2008) The effect of particle design on cellular internalization pathways. Proc Natl Acad Sci USA 105:11613-11618

55. Jacobson-Kram D, Mills G (2008) Leveraging exploratory investigational new drug studies to accelerate drug development. Clin Cancer Res 14:3670-3674

56. Food and Drug Administration/Center for Drug Evaluation and Research (2006) Guidance for industry, investigators and reviewers: exploratory IND studies. http://www.fda.gov/cder/ guidance/7086fnl.htm. Accessed 3 Mar 2008

57. Krestin GP, Bernsen MR (2006) Molecular imaging in radiology: the latest fad or the new frontier? Eur Radiol 16:2383-2385
58. Otero HJ, Erturk SM, Ondategui-Parra $S$ et al (2007) Molecular imaging programs in the United States: results of a national survey. Acad Radiol 14:125-136

59. Kuhn KA, Knoll A, Mewes HW et al (2008) Informatics and medicine. From molecules to populations. Methods Inf Med 47:283-295

60. Dixon AK (2008) Current thoughts about academic radiology. Clin Radiol 63:115-117

61. Hammerstingl R, Huppertz A, Breuer J et al. European EOB-study group. Diagnostic efficacy of gadoxetic acid (Primovist)-enhanced MRI and spiral CT for a therapeutic strategy: comparison with intraoperative and histopathologic findings in focal liver lesions. Eur Radiol 18:457-467

62. United States International Trade Commission (2007) United States International Trade Commission. Medical Devices and Equipment: Competitive Conditions Affecting U.S. Trade in Japan and Other Principal Foreign Markets. http://www.usitc.gov/ext relations/news release/2007/ er0328ee1.htm. Accessed 5 Mar 2008

63. Reinhardt ER (2008) Technical paradigms for realizing ubiquitous care. Stud Health Technol Inform 134:129-134

64. Hillman BJ, Gatsonis C, Schnall MD (2004) The ACR imaging network: a retrospective on 5 years of conducting multicenter trials in radiology and plans for the future. J Am Coll Radiol 1:346350 